\title{
Branching Process Approach to Avalanche Dynamics on Complex Networks
}

\author{
D.-S. LeE, ${ }^{*}$ K.-I. GoH, B. KaHnG and D. KIM \\ School of Physics and Center for Theoretical Physics, Seoul National University, Seoul 151-747
}

(Received 8 October 2003)

\begin{abstract}
We investigate the avalanche dynamics of the Bak-Tang-Wiesenfeld (BTW) sandpile model on complex networks with general degree distributions. With the threshold height of each node given as its degree in the model, self-organized criticality emerges such that the avalanche size and the duration distribution follow power laws with exponents $\tau$ and $\delta$, respectively. Applying the theory of the multiplicative branching process, we find that the exponents $\tau$ and $\delta$ are given as $\tau=\gamma /(\gamma-1)$ and $\delta=(\gamma-1) /(\gamma-2)$ for the degree distribution $p_{d}(k) \sim k^{-\gamma}$ with $2<\gamma<3$, with a logarithmic correction at $\gamma=3$, while they are $3 / 2$ and 2 , respectively, for $\gamma>3$ and when $p_{d}(k)$ follows an exponential-type distribution. The analytic solutions are supported by our numerical simulation results.
\end{abstract}

PACS numbers: 89.70.+c, 89.75.-k, 05.10.-a

Keywords: Avalanche, Complex network

This year the world witnessed a massive power outage plunging a large part of eastern US and neighboring Canadian provinces into darkness, which is known to have affected more than 50 million people [1]. This is one example of global cascades that can always occur in complex systems such as the stock market, the consuming public, the Internet, biological systems, and so on [2]. Such a massive catastrophe sweeping a large part of a system is, however, triggered from only a small fraction of constituents for a not so correspondingly significant reason. Indeed, the authorities examined the cause of the blackout and mentioned that it began with failures of the power transportation system in Ohio [1]. Similarly, some books, movies, and albums become popular hits with small marketing budgets, and occasionally large fluctuations of the stock market cannot be traced to arrival of any significant piece of information. Thus, how such cascades propagate in complex systems is an important problem to understand. It is closely related to the topology of the corresponding networks. Here a complex system is viewed as a network made of nodes and links. A node represents a constituents of the system while a link an interaction between them. What interests us recently is that the degree distributions of complex networks such as the Internet and biological networks [3-5] are power laws, i.e., $p_{d}(k) \sim k^{-\gamma}$, where the degree $k$ of a vertex is the number of links incident on the vertex. The networks following such a power-law degree distribution are called scale-free (SF) networks. Such a slowly-decaying degree distribution implies the presence of a non-negligible fraction of hubs, i.e., nodes of very

*E-mail: dslee@phya.snu.ac.kr large degrees, which would exist with exponentially small probabilities in networks with a Poisson degree distribution, $p_{d}(k) \sim \exp (-\langle k\rangle)\langle k\rangle^{k} / k$ ! or an exponential one, $p_{d}(k) \sim \exp (-k)$. Here, $\langle k\rangle$ denotes the average degree defined as $\langle k\rangle=\sum_{k=1}^{\infty} k p_{d}(k)$. However, the role of these hubs in the cascading propagation is not yet clearly understood.

In this work, we investigate the effect of network topology represented by the degree distribution on the avalanche dynamics in the Bak-Tang-Wiesenfeld (BTW) sandpile [6] model, which was studied extensively on regular lattices as a prototypical system showing selforganized criticality (SOC). In Euclidean space, the main SOC feature of the model is the emergence of a power law in the avalanche size distribution,

$$
p_{a}(s) \sim s^{-\tau} .
$$

Bonabeau [7] has studied the BTW sandpile model on the Erdös-Rényi (ER) random networks and found that the avalanche size distribution follows a power law with the exponent $\tau \simeq 1.5$, consistent with the mean-field solution in Euclidean space [8]. Recently, Lise and Paczuski [9] have studied the Olami-Feder-Christensen model [10] on regular ER networks, where the degree of each node is uniform but connections are random. They found the exponent to be $\tau \approx 1.65$. However, when the degree of each node is not uniform, they found no criticality in the avalanche size distribution. Note that they assumed that the threshold of each node is uniform, whereas the degree is not. While a few studies have been performed on ER random networks, no study of the BTW sandpile model on complex networks with general degree distributions including power-law ones has been performed yet, even though there are several related ap- 
(a)

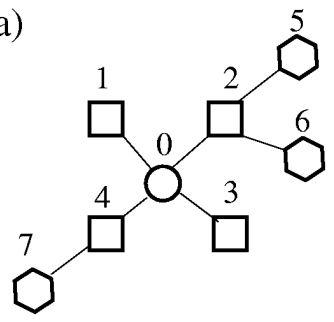

(b)

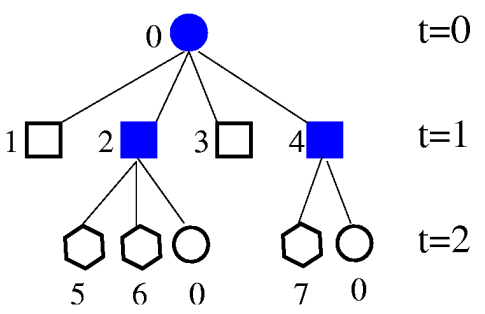

Fig. 1. (a) An avalanche triggered at the node 0 (circle) propagates to the nodes $1,2,3$, and 4 (squares), and then to 5,6 , and 7 (hexagons). No toppling event occurs at the nodes $1,3,5,6$, or 7 . No more than the first toppling occurs at the node 0 . (b) The corresponding tree of the avalanche in (a) can be drawn with three generations $(t=0,1,2)$ depending on the distance from the node 0 , the originator. The avalanche size is 3 , contributed by the nodes, $0,2,4$, denoted by filled symbols.

plications as mentioned above.

We consider the avalanche dynamics on networks with degree distribution $p_{d}(k)$ as follows: (i) At each time step, a grain is added at a randomly chosen node $i$. (ii) If the height at the node $i$ reaches or exceeds a prescribed threshold $z_{i}$, where we set $z_{i}=k_{i}$, the degree of the node $i$, then it becomes unstable and all the grains at the node topple to its adjacent nodes; $h_{i} \rightarrow h_{i}-k_{i}$, and $h_{j}=h_{j}+1$, where $j$ is a neighbor node of the node $i$. (iii) If this toppling causes any of the adjacent nodes to be unstable, subsequent topplings follow on those nodes in parallel until there is no unstable node left. This process defines an avalanche. (iv) Repeat (i)-(iii). We concentrate on the distribution of (a) the avalanche area $A$, i.e., the number of distinct nodes participating in a given avalanche, (b) the avalanche size $S$, i.e., the number of toppling events in a given avalanche, and (c) the duration $T$ of a given avalanche. The study of the avalanche dynamics on SF networks has been carried out in [11]. Here, we keep $p_{d}(k)$ more general than the SF behavior and give more detailed derivation of the results reported in [11].

Analytic solutions for the avalanche size and duration distributions can be obtained by applying the theory of multiplicative branching processes [12]. To each avalanche, one can draw a corresponding tree structure [See Fig. 1]: The node where the avalanche is triggered is the originator of the tree and the branches out of that node correspond to topplings to the neighbors of that node. As the avalanche proceeds, the tree grows. The number of branches of each node is not uniform, but is equal to its own degree. The branching process ends when no further avalanche proceeds. In the tree structure, a daughter node born at time $t$ is located away from the originator by a distance $t$ along the shortest pathway. In the branching process, it is assumed that branchings from different parent nodes occur independently. Then one can derive the statistics of tree size

and lifetime analytically $[8,13]$, which can be considered as that of avalanche size and duration since the avalanche duration $T$ is equal to the lifetime of the tree minus one, and the avalanche size $S$ differs from the tree size only by the number of boundary nodes of the tree, which is relatively small when the overall tree size is very large. The multiplicative branching process approach has been used to obtain the mean-field solution for the BTW model in Euclidean space [8], which is valid above the critical dimension $d_{c}=4$. The avalanches in complex networks usually do not form a loop but have tree-structures: According to the numerical simulations the details of which will be explained later, the probability distributions of the two quantities $A$ and $S$ behave in a similar fashion and, as another example, the maximum area and size $\left(A_{\max }, S_{\max }\right)$ among avalanches are $(5127,5128)$, $(12058,12059)$ and $(19692,19692)$ for networks having the power-law degree distributions with the degree exponents $\gamma=2.01,3.0$, and $\infty$, respectively. $A$ and $S$ being almost the same implies that the avalanche structure can be treated as a tree. From now on, we shall not distinguish $A$ and $S$, and use $s$ to represent either $A$ or $S$.

The branching probability $q(k)$ that a certain node generates $k$ branches in the corresponding tree consists of two factors: One is the probability $q_{1}(k)$ that the node receiving a grain from one of its neighbors has $k$ degrees and the other is the probability $q_{2}(k)$ that toppling indeed occurs at the node. The probability $q_{1}(k)$ is equal to the degree distribution of the node at one end of a randomly chosen edge, i.e., $q_{1}(k)=k p_{d}(k) /\langle k\rangle . q_{2}(k)$ corresponds to the probability that the node has height $k-1$ at the moment of gaining the grain from one of its neighbors. If one assumes that there is no typical height of a node in the inactive state, regardless of its degree $k$, then $q_{2}(k)=1 / k$. Such assumption can be tested numerically: The plot of the probability $q_{2}(k)$ with numerical simulation data supports $q_{2}(k)=1 / k$ as shown in Fig. 2. Deviations from $1 / k$ at large $k$ are presumably due to the finite size effect. Therefore, the branching probability $q(k)$ can be written as

$$
q(k)=q_{1}(k) q_{2}(k)=\frac{p_{d}(k)}{\langle k\rangle} \quad \text { for } \quad k \geq 1,
$$

and $q(0)=1-\sum_{k=1}^{\infty} q(k)$.

Independence of branchings from different parent nodes assumed in the branching process enables one to express the tree size distribution $p(s)$ in terms of the branching probability $q(k)$ as $[12,13]$

$$
p(s)=\sum_{k=0}^{\infty} q(k) \sum_{s_{1}=1}^{\infty} \sum_{s_{2}=1}^{\infty} \cdots \sum_{s_{k}=1}^{\infty} \prod_{i=1}^{k} p\left(s_{i}\right) \delta_{\sum_{i=1}^{k} s_{i}, s-1} .
$$

We define the generating functions, $\mathcal{P}(y)=\sum_{s=1}^{\infty} p(s) y^{s}$ and $\mathcal{Q}(\omega)=\sum_{k=0}^{\infty} q(k) \omega^{k}$ of the tree size distribution $p(s)$ and the branching probability $q(k)$, respectively. 


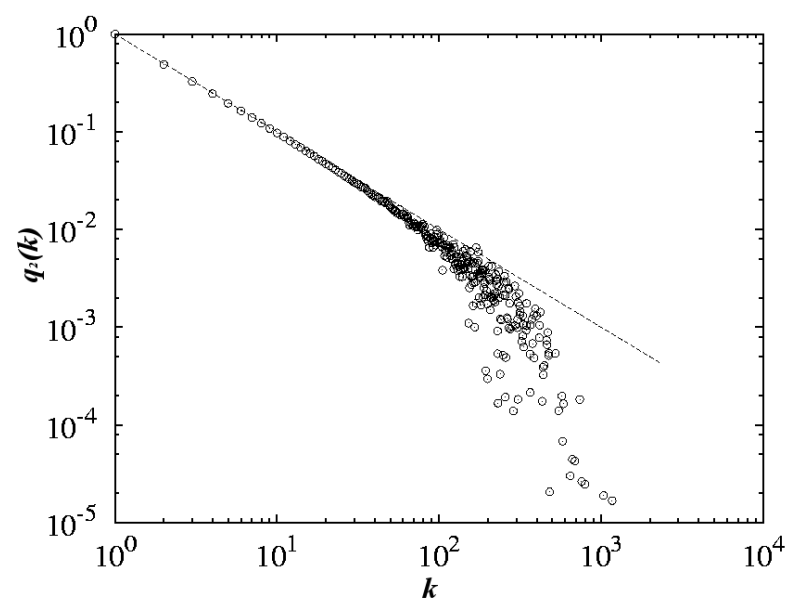

Fig. 2. The probability $q_{2}(k)$ for the degree distribution $p_{d}(k) \sim k^{-3}$. The dashed line represents $1 / k$ Data are for a network of size $N=10^{6}$.

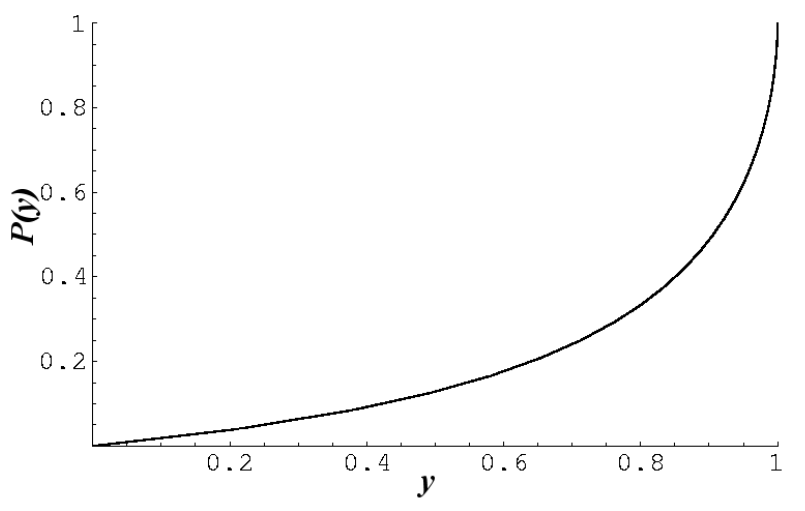

Fig. 3. Plot of $\omega=\mathcal{P}(y)$ for $q(k)=k^{-3.5} / \zeta(2.5)$ obtained by inverting $y=\omega / \mathcal{Q}(\omega)$.

Using Eq. (3), one can show that they are related as follows:

$$
\mathcal{P}(y)=y \mathcal{Q}(\mathcal{P}(y)) .
$$

Then $\omega=\mathcal{P}(y)$ is obtained by inverting $y=\omega / \mathcal{Q}(\omega)$. As an example, the function $\mathcal{P}(y)$ obtained in this way with $q(k)=k^{-3.5} / \zeta(2.5), \zeta(x)$ being the Riemann zeta function, is plotted in Fig. 3.

The distribution of duration, i.e., the lifetime of a tree growth, can be evaluated similarly $[8,12]$. Let $r(t)$ be the probability that a branching process stops at or prior to time $t$. Then, following a similar step to Eq. (3), it is obtained that

$$
r(t)=\mathcal{Q}(r(t-1)) .
$$

For large $t, r(t)$ is close to 1 , and one can obtain $\omega=$ $r(t-1)$ by the approximate relation $d \omega / d t \simeq r(t)-r(t-$ $1)=Q(\omega)-\omega$. The lifetime distribution $\ell(t)$ is just $r(t)-r(t-1) \simeq d \omega / d t$.

The behavior of the generating function $\mathcal{P}(y)$ near $y=$ 1 is related to the asymptotic behavior of the tree size distribution $p(s)$ for large $s$. Consider the case where the function $\mathcal{P}(y)$ has such a non-analytic term as $(1-y)^{\phi}$ with $\phi$ a non-integer, which is expanded as

$$
\begin{aligned}
(1-y)^{\phi} & =\sum_{s=0}^{\infty} a_{s} y^{s}, \\
a_{s} & =\frac{\Gamma[s-\phi]}{\Gamma[s+1] \Gamma[-\phi]} \sim s^{-\phi-1}(s \rightarrow \infty),
\end{aligned}
$$

with $\Gamma(x)$ the Gamma function. Thus, the presence of the non-analytic term $(1-y)^{\phi}$ in $\mathcal{P}(y)$ gives $p(s) \sim s^{-\phi-1}$ for large $s$. On the other hand, the distribution $p(s)$ is expected to decay at least as fast as exponentially if there is no such a non-analytic term.

The value of $\mathcal{P}(1)$ represents the proportion of finite trees among all generated trees, and is used to describe the phase of the process: When $\mathcal{P}(1)$ is smaller than 1 , implying the presence of an infinite tree, the process is in the super-critical phase, while it is in the sub-critical phase when it is equal to 1 . The criterion defines the critical phase. The three phases are also characterized by the average number of daughters defined by

$$
C=\sum_{k=1}^{\infty} k q(k)
$$

The cases of $C<1, C=1$, and $C>1$ correspond to the sub-critical, critical, and super-critical phase, respectively. From Eq. (4), one can notice that in the critical phase, the derivative, $\mathcal{P}^{\prime}(y)$ diverges at $y=1$, implying a non-analytic term $(1-y)^{\phi}$ with $\phi<1$ present in $\mathcal{P}(y)$ and, in turn, the power-law behavior of the tree size distribution. Note that Eq. (2) gives $C=1$ regardless of the degree distribution $p_{d}(k)$, and thus SOC is established generally in the avalanche dynamics on complex networks.

First, we consider the avalanche dynamics on networks with an exponential degree distribution such as $p_{d}(k) \sim$ $\exp (-a k)$ with $a$ a positive constant. The corresponding branching probability given by Eq. (2) is

$$
q(k)= \begin{cases}\exp (-a) & (k=0) \\ \exp [-a(k+1)](\exp (a)-1)^{2} & (k \geq 1),\end{cases}
$$

and its generating function $\mathcal{Q}(\omega)$ is expanded near $\omega=1$ as

$$
\mathcal{Q}(\omega) \simeq \omega+\frac{(1-\omega)^{2}}{\exp (a)-1},
$$

to the leading order in $(1-\omega)$. By inverting the relation $y=\omega / \mathcal{Q}(\omega)$ around $\omega=1$, we obtain that

$$
\mathcal{P}(y) \simeq 1-[(\exp (a)-1)(1-y)]^{1 / 2} .
$$

The tree-size distribution is obtained using Eq. (6) as

$$
p(s) \sim s^{-3 / 2}
$$


for large $s$. Using the generating function $\mathcal{Q}(\omega)$, the duration distribution can also be obtained. The probability distribution $\omega=r(t-1)$ is obtained from

$$
\frac{d \omega}{d t} \simeq \mathcal{Q}(\omega)-\omega=\frac{(1-\omega)^{2}}{\exp (a)-1},
$$

which gives $1-r(t) \sim t^{-1}$ and the duration distribution $\ell(t) \simeq d \omega / d t$ is obtained as

$$
\ell(t) \sim t^{-2}
$$

The exponents $\tau=3 / 2$ and $\delta=2$ in the duration distribution $\ell(t) \sim t^{-\delta}$ are also found in the case of the Poisson degree distribution [13].

Next we consider the power-law degree distribution, $p_{d}(k) \sim k^{-\gamma}$. The branching probability is written as

$$
q(k)= \begin{cases}1-\zeta(\gamma) / \zeta(\gamma-1) & (k=0) \\ k^{-\gamma} / \zeta(\gamma-1) & (k \geq 1)\end{cases}
$$

and its generating function is given by $\mathcal{Q}(\omega)=$ $q_{0}+\operatorname{Li}_{\gamma}(\omega) / \zeta(\gamma-1)$, where $\operatorname{Li}_{\gamma}(\omega)$ is the polylogarithm function of order $\gamma$, defined as $\operatorname{Li}_{\gamma}(\omega)=$ $(\omega / \Gamma[\gamma]) \int_{0}^{\infty}(\exp (y)-\omega)^{-1} y^{\gamma-1} d y$. Contrary to the case of the exponential degree distribution, the generating function $\mathcal{Q}(\omega)$ has a non-analytic term depending on the degree exponent $\gamma$ as [14]

$$
\begin{aligned}
& \mathcal{Q}(\omega)-\omega \simeq \\
& \begin{cases}A(\gamma)(1-\omega)^{\gamma-1} & (2<\gamma<3), \\
-(1 / 2 \zeta(2))(1-\omega)^{2} \ln (1-\omega) & (\gamma=3) \\
(1 / 2) B(\gamma)(1-\omega)^{2} & (\gamma>3)\end{cases}
\end{aligned}
$$

to the leading order in $(1-\omega)$, where $A(\gamma)=\Gamma(1-$ $\gamma) / \zeta(\gamma-1)$ and $B(\gamma)=[\zeta(\gamma-2) / \zeta(\gamma-1)]-1$. From the relation between $\mathcal{Q}(\omega)$ and $\mathcal{P}(y)$ in Eq. (4), we obtain that, for large $s$

$$
p(s) \sim \begin{cases}a(\gamma) s^{-\gamma /(\gamma-1)} & (2<\gamma<3), \\ b s^{-3 / 2}(\ln s)^{-1 / 2} & (\gamma=3), \\ c(\gamma) s^{-3 / 2} & (\gamma>3),\end{cases}
$$

where

$$
\begin{aligned}
a(\gamma) & =-A(\gamma)^{1 /(1-\gamma)} / \Gamma[1 /(1-\gamma)] \\
b & =\sqrt{\pi / 6} \\
c(\gamma) & =\sqrt{1 /(2 \pi B(\gamma))}
\end{aligned}
$$

Thus, the exponent $\tau$ is determined to be $\tau=\gamma /(\gamma-1)$ for $2<\gamma<3$ and $\tau=3 / 2$ for $\gamma \geq 3$. The $\gamma$-dependent generating function $\mathcal{Q}(\omega)$ also causes the duration distribution $\ell(t)$ to have its exponent dependent on $\gamma$, as

$$
\ell(t) \sim \begin{cases}t^{-(\gamma-1) /(\gamma-2)} & (2<\gamma<3), \\ t^{-2}(\ln t)^{-1} & (\gamma=3), \\ t^{-2} & (\gamma>3) .\end{cases}
$$

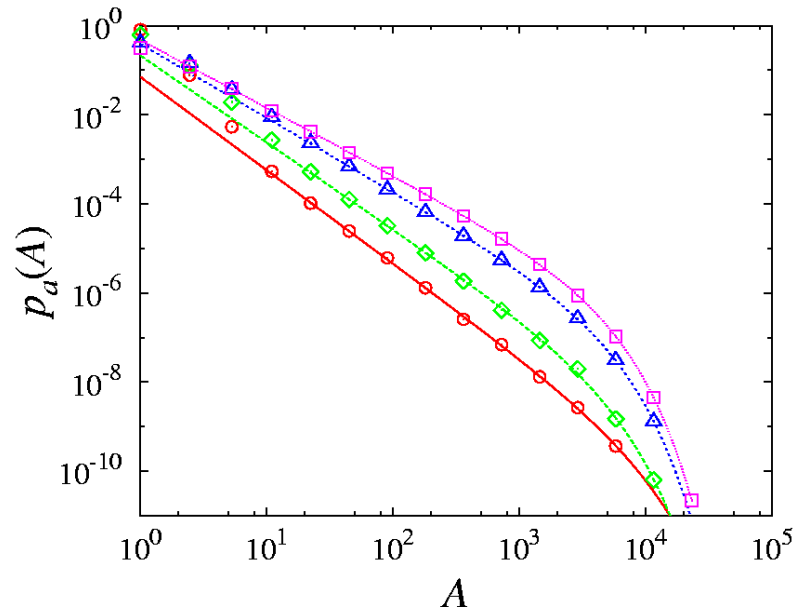

Fig. 4. The avalanche size distributions for the static model of $\gamma=\infty(\square), 3.0(\triangle), 2.2(\diamond)$, and $2.0(\bigcirc)$. The data are fitted with the form $p_{a}(A) \sim A^{-\tau} \exp \left(-A / A_{c}\right)$ with $A_{c}$ a cutoff due to the finite size effect. For the fitted values of $\tau$, see Table 1 . Data are logarithmically binned.

Table 1. Values of the avalanche size exponent $\tau$ and duration exponents $\delta$ for the static model with mean degree 4 and of size $N=10^{6}$. The subscripts $m$ and $t$ mean the measured and theoretical values, respectively. Note that since the duration exponent diverges theoretically as $\gamma \rightarrow 2$, numerical simulation data contain lots of fluctuations from sample to sample. ${ }^{*}$ The case of $\gamma=3$ has logarithmic corrections in $\tau_{t}$ and $\delta_{t}$.

\begin{tabular}{ccccc}
\hline \hline$\gamma$ & $\tau_{m}$ & $\tau_{t}$ & $\delta_{m}$ & $\delta_{t}$ \\
\hline$\infty$ & $1.52(1)$ & 1.50 & 1.9 & 2 \\
5.0 & $1.52(3)$ & 1.50 & 2.0 & 2 \\
$3.0^{*}$ & $1.66(2)$ & 1.50 & 2.5 & 2 \\
2.8 & $1.69(3)$ & 1.56 & 2.6 & 2.25 \\
2.6 & $1.75(4)$ & 1.63 & 2.9 & 2.67 \\
2.4 & $1.89(3)$ & 1.71 & 3.5 & 3.50 \\
2.2 & $1.95(9)$ & 1.83 & 4.3 & 6.00 \\
2.01 & $2.09(8)$ & 2.0 & & $\infty$ \\
\hline \hline
\end{tabular}

That is, the exponent $\delta$ is given by $\delta=(\gamma-1) /(\gamma-2)$ for $2<\gamma<3$ and $\delta=2$ for $\gamma \geq 3$.

To check the analytic solutions, we performed numerical simulations for SF networks in the static model [15], since nontrivial values of exponents $\tau$ and $\delta$ are expected to be observed in networks with power-law degree distributions as seen in Eqs. (16) and (18). Additionally, we allow a small fraction $f=10^{-4}$ of grains to be lost in the simulations, to prevent the system from being overloaded in the end. The avalanche area distribution shown in Fig. 4 gives the exponent $\tau$ dependent on $\gamma$ when fitted with the form $p_{a}(A) \sim A^{-\tau} \exp \left(-A / A_{c}\right)$ with $A_{c}$ as a cutoff. We have also considered the avalanche duration distribution. However, the duration distribution is not well shaped numerically in finite size systems, since the 
duration of an avalanche does not run long enough, due to the small-world effect. So, we measure the dynamic exponent $z$ defined by $s \sim t^{z}$ and then evaluate the duration exponent $\delta$ from the relation $p(s) d s \sim \ell(t) d t$ or $\delta=1+(\tau-1) z$. The numerical values of $\tau$ and $\delta$ for different $\gamma$ are tabulated in Table 1 and show reasonable agreement with those obtained analytically. The discrepancy can be attributed to the finite-size effect, as well as the characteristic avalanche size set by the probability of losing a grain $\left(f=10^{-4}\right)$.

In conclusion, we have studied the BTW sandpile model on complex networks with general degree distributions. To account for the heterogeneity of the system, the threshold height of each vertex is set to be equal to the degree of the vertex. By mapping to the multiplicative branching process, we can obtain the asymptotic behaviors of the avalanche size and duration distributions analytically. The avalanche size and duration exponents $\tau$ and $\delta$ deviate from their conventional mean-field values $\tau=3 / 2$ and $\delta=2$ when the degree exponent $\gamma$ is smaller than 3. The result remains the same if the threshold contains noise such as $z_{i}=\eta_{i} k_{i}$, with $\eta_{i}$ being distributed uniformly in $[0,1]$ and when a new grain is added to a node chosen with probability proportional to the degree of that node. Following the same steps, we can also obtain the exponents $\tau$ and $\delta$ for the more general case where $z_{i}=k_{i}^{\beta}$ with $0<\beta \leq 1$. Then $\tau=(\gamma+2 \beta-2) /(\gamma+\beta-2)$ and $\delta=(\gamma+\beta-2) /(\gamma-2)$ for $2<\gamma<\gamma_{c}$ where $\gamma_{c}=\beta+2 \leq 3$, and $\tau=3 / 2$ and $\delta=2$ for $\gamma>\gamma_{c}$. The fact that $\tau$ increases as $\gamma$ decreases implies that the hubs sustain large numbers of grains, explaining the resilience of the network under avalanche phenomena. This is reminiscent of the extreme resilience of the network under random removal of vertices for $\gamma \leq 3 \quad[5,16,17]$.

\section{ACKNOWLEDGMENTS}

This work is supported by the KOSEF Grant No. R142002-059-01000-0 in the ABRL program.

\section{REFERENCES}

[1] http://abcnews.go.com/ (2003).

[2] D.J. Watts, Proc. Natl. Acad. Sci. USA 99, 5766 (2002).

[3] R. Albert and A.-L. Barabási, Rev. Mod. Phys. 74, 47 (2002).

[4] S. N. Dorogovtsev and J. F. F. Mendes, Adv. Phys. 51, 1079 (2002).

[5] R. Albert, H. Jeong, and A.-L. Barabási, Nature (London) 406, 378 (2000).

[6] P. Bak, C. Tang and K. Wiesenfeld, Phys. Rev. Lett. 59, 381 (1987); Phys. Rev. A 38, 364 (1988).

[7] E. Bonabeau, J. Phys. Soc. Jpn. 64, 327 (1995).

[8] P. Alstrøm, Phys. Rev. A 38, 4905 (1988).

[9] S. Lise and M. Paczuski, Phys. Rev. Lett. 88, 228301 (2002)

[10] Z. Olami, H. J. S. Feder and K. Christensen, Phys. Rev. Lett. 68, 1244 (1992); K. Christensen and Z. Olami, Phys. Rev. A 46, 1829 (1992).

[11] K.-I. Goh, D.-S. Lee, B. Kahng and D. Kim, Phys. Rev. Lett. 91, 148701 (2003).

[12] T. E. Harris, The Theory of Branching Processes (Springer-Verlag, Berlin, 1963).

[13] R. Otter, Ann. Math. Statist. 20, 206 (1949).

[14] J. E. Robinson, Phys. Rev. 83, 678 (1951).

[15] K.-I. Goh, B. Kahng and D. Kim, Phys. Rev. Lett. 87, 278701 (2001).

[16] R. Cohen, K. Erez, D. ben-Avraham and S. Havlin, Phys. Rev. Lett. 85, 4626 (2000).

[17] D. S. Callaway, M. E. J. Newman, S. H. Strogatz and D. J. Watts, Phys. Rev. Lett. 85, 5468 (2000). 